\title{
Quercetin Induces Lipid Domain-Dependent
}

\section{Permeability}

Natália B. Leite*łf, Danubia B. Martins§, Marcia P. dos Santos Cabrerał

†Departamento de Química e Ciências Ambientais and §Departamento de Física, Universidade Estadual Paulista (Unesp), Instituto de Biociências Letras e Ciências Exatas (Ibilce), Câmpus São José do Rio Preto, SP, Brazil.

*corresponding author

\section{KEYWORDS}

quercetin; cholesterol; lipid bilayer effects; giant vesicles microscopy; phospholipid membranes; lipid/water partition coefficients;

ABSTRACT: Quercetin is a polyphenolic molecule that has a broad spectrum of biological activities derived from its antioxidant property. Its mechanism of action has been explained by their binding and/or interference with enzymes, receptors, transporters and signal transduction systems. Since these important mechanisms generally occur in membrane environments, within and through lipid bilayers, investigating the biophysical properties related to the diversity of lipid compositions of cell membranes may be the key to understanding the role of cell membrane in these processes. In this work, we explored the interaction of quercetin with model membranes of different lipid compositions to access the importance of lipid phases, $l d$, $l d / l o$ and $l d+l o+s o$, to the action of quercetin in bilayers and possibly contribute to the understanding of quercetin multiple activities. Analysis of the influence of quercetin on the morphology and permeability of GUVs, rigidity of LUVs and affinity to these vesicles showed that quercetin strongly partitions to the more 
homogeneous environments, but strongly permeates and modifies the more heterogeneous $l d+l o+s o$ vesicles (PC/SM/Chol). The rigidifying effect of Chol at $40 \%$ was enhanced by quercetin, while in the PC/SM/Chol membranes, a dual behavior was observed: bilayers became more flaccid at low quercetin/lipid proportions $(<1 / 5)$ and moderately rigid at proportions of the $1 / 1$ order. Quercetin is an important flavonoid for its high antioxidant properties and our results shed new light to quercetin effects on lipid bilayers, and help to understand its wide range of physiological effects.

\section{INTRODUCTION}

Natural polyphenols are antioxidants widely distributed in plants, ubiquitous in the human diet, present at small concentration, but especially important to inhibit lipid peroxidation, a serious degenerative process in cells. The high affinity of flavonoids for membranes is responsible for their rapid, localized concentration. The membrane concentrated presence of these antioxidants is related to a full repertoire of activities in the prevention of oxidative processes as those occurring in coronary heart diseases, cancer and age-dependent neuropathologies. ${ }^{1}$

The polyphenol quercetin, is a flavonoid with antioxidant properties attributed to the presence of 5 hydroxyl groups in the structure $\left(3,3^{\prime}, 4^{\prime}, 5,7\right.$-pentahydroxyflavone) bound to aromatic rings and forming delocalized phenoxyl radicals within the structure. ${ }^{1}$ Among quercetins's biological activities, there is evidence for protection against cardiovascular disease, anticancer and antiviral effects ${ }^{2,3}$, as well as antihypertensive and renal protective effects ${ }^{4}$ and protection against mitochondrial dysfunction, oxidative stress and inflammation induced by cholesterol. ${ }^{5}$

Important for quercetin antioxidant activity are the orientation in the membrane lipid bilayer, the affinity and the distribution. ${ }^{6}$ The oil/water partition coefficient of $1.8(\log \mathrm{P}$ for curcumin $=3$ ) indicates the quercetin preference for the membrane environments ${ }^{3}$, moreover, also shown to selectively prefer the membrane interface in relation to hydrophobic phases. ${ }^{7}$ Indeed, at physiological $\mathrm{pH}$, quercetin interacts with polar head groups at the water/lipid interface. ${ }^{8}$ This partitioning of quercetin into the bilayer involves electrostatic interactions, as hydrogen bonds between hydroxyl groups and polar groups of phospholipids, hydrophobic interactions with the acyl chains and is affected by the geometry of phospholipids. ${ }^{6}$

Quercetin molecules are considered comparable to cholesterol in relation to location and 
influence in the lipid bilayer. ${ }^{6,9}$ Data obtained through anisotropy of fluorescent probes indicate that anisotropy increases with quercetin concentration, indicative of a rigidifying effect on the bilayer $^{2,6,10}$ as observed with bilayers containing cholesterol. ${ }^{11}$ This rigidifying effect of quercetin was correlated with anticancer and antibacterial activities. ${ }^{2,10}$ Additionally, calorimetry studies (DSC) indicate a condensation effect, which was attributed to the flat structure of quercetin enabling it to intercalate between the hydrocarbon chains. ${ }^{9}$ Moreover, this latter work also suggests that quercetin preferentially interacts with some lipids, segregating raft-like $l_{o}$ lipid domains which are surrounded by $l_{d}$ lipids. One of these lipids would be sphingomyelin whose long acyl chains could make space for quercetin interdigitation besides favoring polar interactions. ${ }^{9}$ These effects, responsible for higher heterogeneity in the membrane, lead to defects in their barrier property, increasing the permeability. ${ }^{10}$

Recently, De Granada-Flor and colleagues ${ }^{12}$ investigated the interactions of quercetin with biologically relevant membrane lipid bilayers. They compared its action in lipid compositions in the $l o$ and $l d$ phases by observing high partition with low interference in the more fluid membrane domains, but strong perturbation of the chol/sphingolipid enriched ones. The lipid composition PC:SM:Chol is characteristic of plasma membrane outer leaflet and widely described in the literature as lipid domain composition where different lipid phases may coexist. ${ }^{13,14}$ The presence of these structures is able to alter the structural and physical parameters of the lipid membrane as we show in the present work. We investigate the role of lipid compositions of higher and lower homogeneity where different lipid phases were represented, as $l d, l d / l o$ and $l d+l o+s o$ on the interaction with quercetin. We analyzed the influence of quercetin on the morphology and permeability of GUVs, on the rigidity of LUVs and the affinity to these vesicles. We found that quercetin strongly partitions to the more homogeneous environments, but strongly permeates and modifies the more heterogeneous $l d+l o+s o$ vesicles (PC/SM/Chol). The rigidifying effect of Chol at $40 \%$ was enhanced by quercetin, while in the PC/SM/Chol membranes, a dual behavior was observed: the bilayers became more flaccid at low quercetin/lipid proportions $(<1 / 5)$ and moderately rigid at proportions of the $1 / 1$ order. Quercetin is an important flavonoid for its high antioxidant properties and studies with model plasma membranes are important to understand its multitude of biological activities, including membrane-related signalling effects, as well as to foresee new applications.

\section{RESULTS}

\subsection{Quercetin Induces Lipid Domain-Dependent Leakage}


To study the role of distinct lipid composition, relevant for mammalian cell membranes ${ }^{15,16}$, on the quercetin activity, GUVs composed by PC, PC/Chol (80:20 and 60:40) and PC/SM/Chol 40:40:20 were prepared and observed through phase contrast and fluorescence microscopies. The experiment allows real time observation of changes in vesicle morphology and in phase contrast intensity as a consequence of quercetin interaction. Examples of these observations are depicted in Figure $1 \mathrm{~A}-\mathrm{D}$ and corresponding control experiments are shown in Figure S1, in which for each lipid composition vesicles were diluted in buffer and observed at the same conditions. This control showed that GUVs were stable, circular structures, whose phase contrast intensity was kept unchanged throughout the observation period regardless of the lipid composition.

In general, all lipid compositions exhibited events of GUV brightness reduction as a result of sucrose leakage. Despite this, the structure of the GUVs and their average diameters were maintained. This membranolytic behavior was observed in all lipid compositions, but the frequency of these events was shown to be lipid composition-dependent. The analysis of the occurrence of these events showed that $38 \%$ and $35 \%$ of vesicles, respectively made of pure PC and of PC/Chol 80:20, lost phase contrast during the observation time lapse. Meanwhile, for the composition with double cholesterol content, PC/Chol 60:40, just 15\% of vesicles lost phase contrast, indicating that increased Chol presence impairs sucrose leakage. In contrast, the presence of SM increased the frequency of sucrose leakage reaching $65 \%$ of the observed vesicles.

This particular behavior in relation to the other lipid compositions might be attributed to the fact that the composition PC/SM/Chol 40:40:20 is characteristic of phase coexistence presenting lipid domains $^{13,14,17}$, what may be influencing the quercetin-lipid interaction. To explore further these observations, GUVs containing fluorescent probes were also visualized for 30 minutes by fluorescence microscopy under quercetin interaction. Control experiments, carried out in the absence of quercetin, had already shown that the lipid compositions PC (100\%) and PC/Chol (80:20 and 60:40) present uniformly bright images of vesicles, while the presence of SM results in the coexistence of brighter and darker circular regions on the vesicles surface, showing that the composition PC/SM/Chol (40:40:20) exhibits microscopic lipid domains (Figure S1). Fluid phase domains display circular shape to minimize line tension and may coalesce upon contact as well as bud out. ${ }^{18}$ Nevertheless, bud outs were not observed in the experimental conditions of the control tests. Fluorescence images from PC/SM/Chol (40:40:20) GUVs show that quercetin contributes to the coalescence of lipid domains, so that its initial configuration is altered by reducing the amount of these structures and increasing their areas on the vesicles surface. At the end of the observation, the vesicles remain with larger dark and shiny areas in comparison to those observed in the initial images. This entire process was accompanied by leakage events and formation of denser regions 
around the boundaries of vesicles could be observed in fluorescence and phase contrast images. The observation of denser regions is not exclusive to the composition $\mathrm{PC} / \mathrm{SM} / \mathrm{Chol}$, but it is common to those containing Chol (Figure 1). Figures 1 B1, B2 and D1, D2 show these dense regions, as bright spots, in fluorescence images recorded at different focal planes.

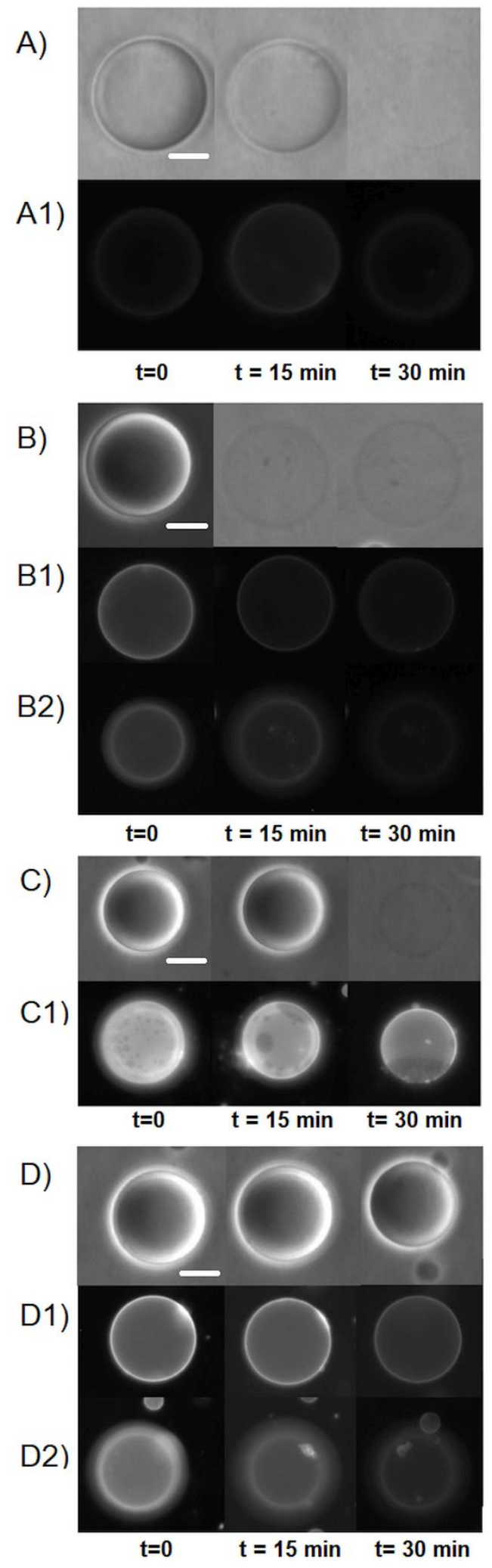


Figure 1. GUV Microscopy. Phase contrast and fluorescence images of quercetin-lipid interactions during $30 \mathrm{~min}$ observation. The observation chamber of $120 \mu \mathrm{L}$ volumetric capacity was prepared with $1 \mu \mathrm{L}$ GUVs suspension in buffer and $21.5 \mu \mathrm{mol} \mathrm{L}^{-1}$ quercetin solution. A) PC (100\%) phase contrast and respective A1) fluorescence microscopy images of GUVS labeled with NBD-PE; B) PC/Chol (80:20) phase contrast and respective B1) fluorescence microscopy images; B2) fluorescence microscopy images under different focal planes to emphasize domain formation; C) PC/SM/Chol (40:40:20) phase contrast and respective C1) fluorescence microscopy images; D) PC/Chol (60:40) phase contrast and respective D1) fluorescence microscopy images; D2) fluorescence microscopy images under different microscope focus. Chol containing GUVs were labeled with TopFluor-Chol. The experiments were performed at $25{ }^{\circ} \mathrm{C}$ and $\mathrm{pH} \mathrm{7.0.} \mathrm{The} \mathrm{bar} \mathrm{is}$ equivalent to $10 \mu \mathrm{m}$.

\subsection{Vesicles permeability to quercetin is cholesterol concentration dependent}

The phase contrast loss induced by quercetin on GUVs was accompanied along time. The images were analysed to describe the sucrose leakage patterns at each experimental condition. Figure 2 shows this analysis. These results emphasize the differences between the leakage events due to SM and Chol presence. Figure 2A shows for one vesicle of each composition that at the beginning of observation there are constant average pixel intensities representing the time during which GUVs rely intact and bright. This constancy is interrupted when the leakage process begins and may occur abruptly or gradually, representing the average permeability $(\mathrm{nm} / \mathrm{s})$, until it reaches a plateau representing that the system reaches an equilibrium condition. PC/SM/Chol vesicles showed the most abrupt leakage profiles followed by PC (100\%), PC/Chol (80:20) and (60:40) and exhibiting the same tendency as the frequency of leakage events. The average time for the leakage to begin, $\left\langle t_{L}\right\rangle$, was also considered to evaluate the leakage process whose values for each lipid composition are shown in Table 1. Results of $\left\langle\mathrm{t}_{\mathrm{L}}\right\rangle$ show that the leakage process begins on average more quickly for the compositions PC and PC/Chol (80:20) followed by PC/Chol (60:40) and PC/SM/Chol. In the presence of SM, there is a delay on the effects of the quercetin-membrane interaction, which could be related to the coalescence of microdomains observed through fluorescence microscopy. The decreasing of $\left\langle\mathrm{t}_{\mathrm{L}}\right\rangle$ from $\mathrm{SM}$ vesicles to $40 \% \mathrm{Chol}$, to $20 \%$ Chol, to $0 \%$ Chol vesicles suggests that an increasing homogeneity and/or decreasing rigidity of bilayers may affect the beginning of the leakage process as discussed below. ${ }^{17,19}$ Examples of the calculation method for average permeability are shown in Figure 2B and the results are expressed in Table 1. These results show that in relation to PC and PC/Chol (80:20) the presence of SM increased the vesicles average permeability in an order of magnitude, while double Chol content reduced it by an order of 
magnitude. This behavior is also expressed by the distribution of GUVs permeabilities (Figure 2C), where the opposite effects of doubling Chol content or substituting PC by SM can be noticed.

Considering that POPC is a low $\mathrm{T}_{\mathrm{M}}$ lipid and its presence in binary and ternary mixtures with Chol and/or SM might result in distinct lipid phase coexistence, the results obtained so far can be addressed under the light of these elements. The binary mixtures fall within the $l d / l o$ region on the phase diagram obtained by Almeida and coll. at $23^{\circ} \mathrm{C} .{ }^{17}$ The coexistence of both, liquid disordered and liquid ordered phases, implies the existence of less homogeneous regions since these regions, present in the bilayer, must accommodate in some way. A few bright dots could be seen in the GUVs fluorescence microscopy at different focal planes, more frequently observed in PC/Chol (60:40) (Figure 1B2 and 1C2). This raises the question that these binary mixtures may lead to the formation of nanoscopic lipid domains and the literature reports the difficulty in detecting them by conventional microscopy. ${ }^{13,20-22}$ Direct observation at nanoscale in binary supported lipid bilayers with POPC showed homogeneous phases in the presence of cholesterol at 25 and $33 \mathrm{~mol} \%$, while domains of about $100 \mathrm{~nm}$ radii were characterized at $50 \mathrm{~mol} \% \mathrm{Chol}$ and $24{ }^{\circ} \mathrm{C} .{ }^{19}$ Considering that the sole presence of bright dots does not characterize lipid domains, the findings of Sarangi et. al ${ }^{19}$ support the similarities of behavior we found for PC (100\%) and PC/Chol (80:20), since these compositions can present the highest homogeneity degrees. The $40 \% \mathrm{Chol}$ mixture displays $l d / l o$ coexistence with regions where lipid packing tends to be more ordered than the $20 \%$ Chol mixture. This is in agreement with the reduced frequency of leakage events and membrane permeability that were found due to the increase in Chol content. However, the ternary mixture is located in the $l d+l o+s o$ region ${ }^{17}$, which gave rise to the observation of lipid domains on a micrometric scale, and corresponding to a bilayer of lower homogeneity that intensified the quercetin membranolytic activity. It is important to consider that in this condition, it is still possible to have the coexistence of domains in nano- and micro metric scales since the presence of one does not exclude the presence of the other. ${ }^{23}$ Lastly, if we notice that the differences between PC/Chol (80:20) and the ternary mixture are the SM presence and the $\mathrm{T}_{\mathrm{M}}$ of lipids (low for PC $v s$ high for SM), they took the system to a condition where the difference in the homogeneity of the bilayer affected the membrane barrier characteristics as evidenced by a more intense leakage. 

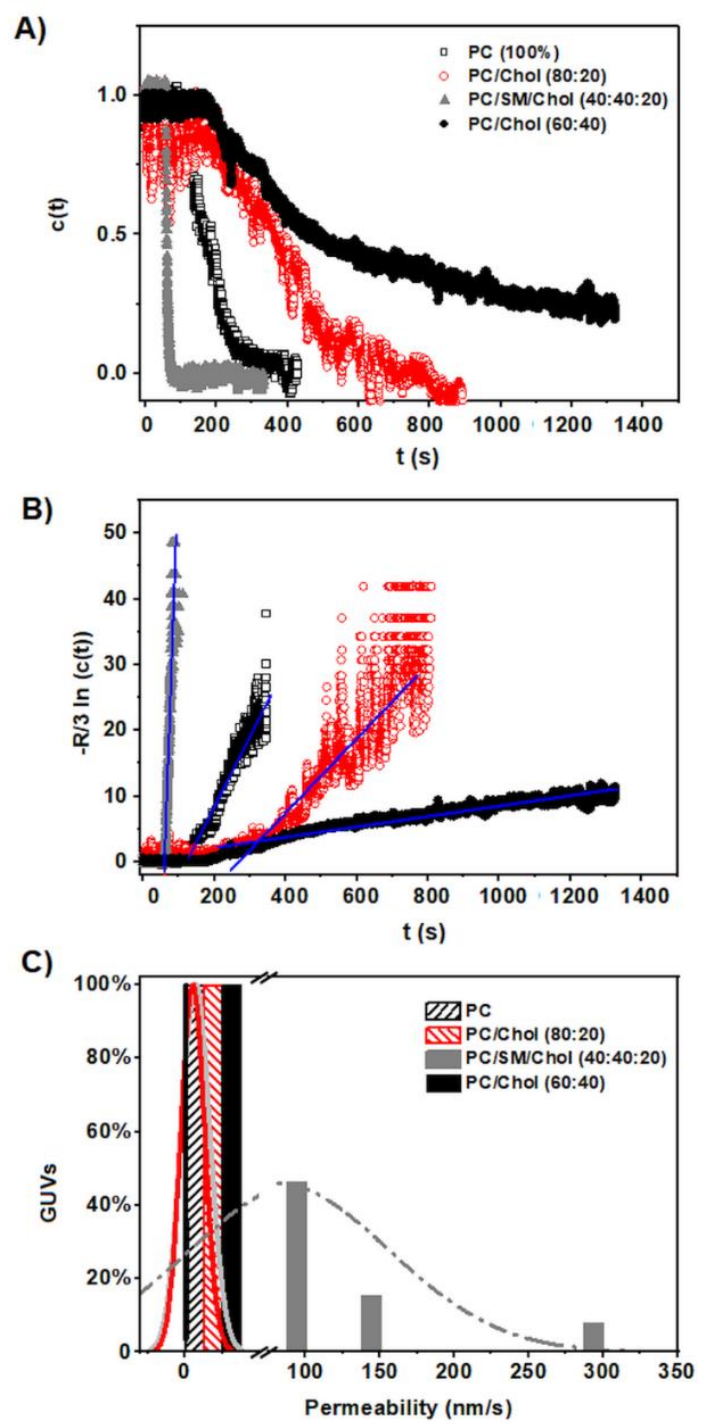

Figure 2. Permeability data. A) Example of sucrose leakage profile acquired from pixel intensities after analysis by ImageJ Software of the phase contrast time lapse data. B) Illustrative graphic calculation of GUVs permeability induced by quercetin during the time lapse in the phase contrast experiments. Blue lines represent linear fit from which slopes result on permeability parameters. C) Distribution of individual GUV permeability results for each lipid composition. Lines represent the statistical Gaussian distribution evidencing the average results. 
Table 1. Average permeability induced by quercetin on distinct lipid bilayers, average time for the leakage to begin, $\left\langle t_{L}\right\rangle$, and the respective number of vesicles observed, $\mathrm{N}$.

\begin{tabular}{l|ccc} 
& $\begin{array}{c}\text { Average permeability } \\
(\mathbf{n m} / \mathbf{s})\end{array}$ & Average $<\mathbf{t}_{\mathbf{L}}>(\mathbf{s})$ & $\mathbf{N}$ \\
\hline PC & $42.0 \pm 21.4$ & $262 \pm 44$ & 8 \\
PC/Chol (80:20) & $33.6 \pm 18.0$ & $291 \pm 48$ & 7 \\
PC/SM/Chol (40:40:20) & $478.8 \pm 126.0$ & $569 \pm 130$ & 13 \\
PC/Chol (60:40) & $5.4 \pm 0.2$ & $336 \pm 67$ & 3
\end{tabular}

\subsection{Chlorophyll emission anisotropy shows that Quercetin stiffens bilayers with 40\% Chol.}

The fluorescent probe chlorophyll a, added to the lipid mixtures in LUVs preparation, reports changes in lipid packing order induced by the quercetin-lipid interaction. ${ }^{6}$ Chlorophyll a locates its fluorescent macrocycle group at the polar headgroups region of the lipid bilayer, at the water interface, and its phytol chain in the acyl chain region. ${ }^{6}$ Increased acyl chain order can be related to increased average fluorescence emission anisotropy values, while the reduction of these values is related to a less ordered packing. ${ }^{24}$ Figure 3 shows the results obtained for increasing quercetin concentration. In the absence of quercetin, $r$ values are characteristic of the LUVs lipid composition and correspond to the fluidity order of each lipid bilayer; $r=0.030 \pm 0.001$ for PC, $r=0.034 \pm$ 0.002 for PC/Chol 80:20, $r=0.036 \pm 0.001$ for PC/SM/Chol 40:40:20, and $r=0.041 \pm 0.001$ for PC/Chol 60:40. The presence of increasing Chol contents increased the average $r$ values. Chol increases membranes' stiffness by decreasing molecular free-volumes and increasing the order of the lipid acyl chains. ${ }^{25}$ When quercetin was added, $r$ values generally increased with the concentration for all lipid compositions tested. However, $\Delta r$ values were smaller for PC and $\mathrm{PC} / \mathrm{SM} / \mathrm{Chol} 40: 40: 20,+0,007$ and $+0,006$, respectively, considering the highest quercetin concentration, 25 and $50 \mu \mathrm{mol} \mathrm{L} \mathrm{L}^{-1}$. For the intermediary concentrations, $r$ in PC showed a tendency to higher values, especially at concentrations $<10 \mu \mathrm{mol} \mathrm{L}^{-1}$, while in PC/SM/Chol the tendency was to lower $r$ values. For PC/Chol vesicles maximum $\Delta r$ values were $+0,029$ in PC/Chol 60:40 and $+0,009$ in PC/Chol 80:20. The highest $r$ value obtained in PC/Chol 60:40, indicative of the highest packing order and a stiffened bilayer, corresponds to the lowest estimated permeability (Table 1). Contrasting, PC/SM/Chol (40:40:20) exhibited an initial fluctuation followed by modest $r$ increase suggesting that quercetin is able to reduce somewhat the lipid packing order under lower quercetin/lipid molar ratio. Earlier studies with curcumin showed its ability to steadily decrease membrane rigidity in a range of lipid compositions including the presence and absence of SM and Chol among other lipids, with exception of vesicles containing $40 \%$ Chol. ${ }^{11}$ Shortly, either quercetin or curcumin increase lipid packing order when $40 \%$ Chol is present. Again, the 
differences between PC/Chol (80:20) and the ternary mixture emphasize the role of SM in leading to the highest average permeability of a more flaccid membrane, more prone to the passage of water and solute molecules under quercetin influence.

The comparison of the results of PC and PC/Chol (80:20 and 60:40) give rise to a discussion about the role of cholesterol in the interaction of quercetin with these membranes. Our results show the intermediate situation of PC/Chol (80:20) where lo/ld phases coexist, and in this homogeneous system $^{19}$ quercetin promotes a limited increase in lipid packing order. However, at $40 \%$ Chol in the mixture, the effect of quercetin increasing membrane stiffness becomes more pronounced. Thus, quercetin increases the bilayer rigidity at $40 \%$ Chol content as curcumin does, but at $20 \%$ Chol content quercetin shows an increase in the bilayer rigidity while curcumin shows the opposite. ${ }^{11}$

Ingólfsson et al. ${ }^{26}$ found that besides curcumin other phytochemicals are located in the bilayer/solution interface, suggesting a common mechanism that seems to be valid for quercetin as well. ${ }^{27}$ In the absence of Chol, quercetin fluidizes the membrane and interacts with the hydrophilic head groups of lipids. Once hydrogen bonds are formed between these polar head groups and the hydroxyl groups of quercetin, they have the effect of posing a barrier to quercetin entry deep into the hydrophobic part of the membranes. ${ }^{27}$ In this sense, as quercetin is able to establish more hydrogen bonds than curcumin due to the presence of more hydroxyl groups this may lead to a more superficial interaction with the lipid bilayer. Our results showed that quercetin made the POPC membrane more flaccid at 1:20 quercetin:lipid molar ratio, what is not observed above the 1:10 ratio when quercetin tends to moderately rigidify the lipid bilayer in agreement with de Granada-Flor et al. ${ }^{12}$ and with the condensing effect at 1:1 quercetin/lipid ratio observed in DSC experiments by Tarahovsky et al. ${ }^{9}$. De Granada-Flor et al. ${ }^{12}$ also performed molecular dynamic simulations in POPC and POPC/Chol (1:1) bilayers showing that quercetin presents a deeper penetration in the Chol absence. We suggest that the presence of Chol hinders the interaction of quercetin, reducing its consequences, as indicated by the microscopy data. Furthermore, at $25^{\circ} \mathrm{C}$, POPC is in the liquid disordered phase and quercetin accommodates easier in systems enriched in this lipid than in a more ordered system as those containing Chol and exhibiting the coexistence of $l d$ and lo phases. 


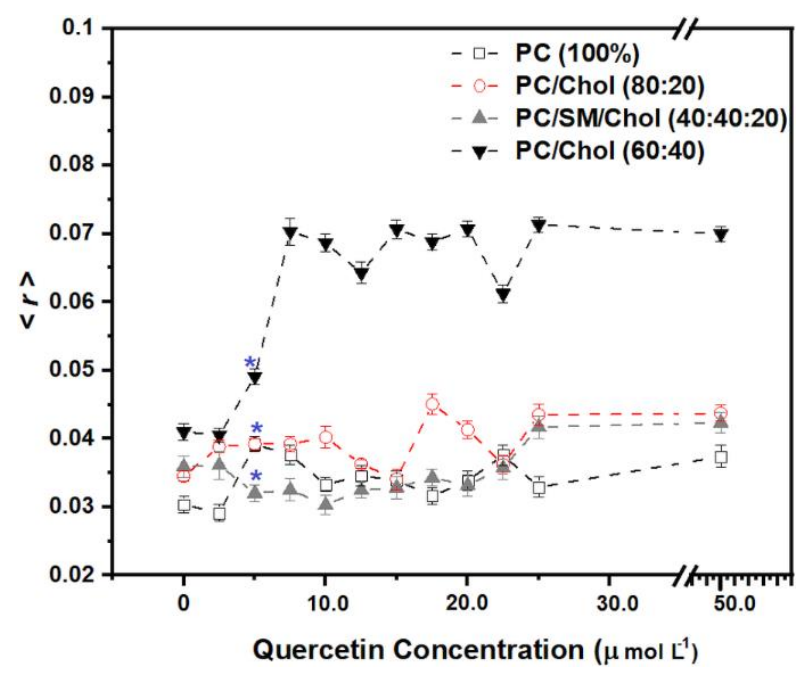

Figure 3. Chlorophyll an emission anisotropy. Changes on chlorophyll an emission anisotropy induced by increasing concentrations quercetin. Lipid concentration $50 \mu \mathrm{mol} \mathrm{L}{ }^{-1}$. Blue stars are relative to de Granada - Flor et al. (2019).

\subsection{Affinity parameters of quercetin to phospholipid bilayers.}

The affinity of quercetin for phospholipid bilayers was evaluated through spectrophotometric titrations and through the steady state emission anisotropy of quercetin. Molecular lipid/water partition coefficients $\left(K_{P}\right)$ in vesicles were determined considering the effects of Chol levels, at 0, 20 and 40\% and the presence of 20\% SM. Figure 4A shows quercetin spectra obtained in the absence (in buffer) and in the presence of the different vesicles. Changes on the quercetin absorbance spectra were registered at each lipid addition. In the absence of lipid, the maximum wavelength was exhibited at $372 \mathrm{~nm}$, but as soon as LUVs suspension was added, increasing intensity on the spectra were observed. Figure S2 exemplify these changes for PC (100\%) lipid composition. In all lipid compositions quercetin showed increased absorbance as a function of lipid addition. Additionally, shifts to higher wavelengths were observed as a consequence of quercetin-lipid interaction, reaching a maximum redshift change of $9 \mathrm{~nm}$ for PC (100\%) and a minimum of $5 \mathrm{~nm}$ for PC/SM/Chol (40:40:20) (Figure 4B). The increased absorbance and the bathochromic effect correspond to quercetin moving from an aqueous environment to a more hydrophobic one. ${ }^{3}$

The determination of $K_{P}$ is illustrated in Figure 4C, where an isotherm curve is plotted showing the normalized absorbance intensities as a function of lipid concentration, and then adjusted by a non-linear fit according to eq. $2 .{ }^{28}$ Table 2 shows the partition coefficients obtained for each lipid composition. These results showed that quercetin partitions into the lipid bilayers in a lipid composition-dependent way. Similarly, to curcumin ${ }^{11}$, Chol at $20 \%$ does not reduce quercetin 
partitioning in relation to $\mathrm{PC}$ vesicles. Nevertheless, $K_{P}$ values are reduced at $40 \% \mathrm{Chol}$ and in the presence of $20 \% \mathrm{SM}$, which correlate to the fact that higher ordered lipid bilayers reduce $K_{P}$ values when compared to the less ordered ones. ${ }^{29}$

The determination of $K_{P}$ was also performed through changes on quercetin emission anisotropy to confirm absorptiometry results. Under lipid titration, quercetin tends to increase anisotropy values in all analysed compositions (Figure 4D). This result is a consequence of quercetin-lipid interaction and reflects the loss of the rotational diffusion of the flavonoid bound to the lipid phase. Chol content showed to be a strong influencer on the anisotropy changes. In addition to causing an increase in lipid packing, in the presence of $40 \%$ Chol the affinity of quercetin was confirmed to be significantly reduced and the absence of Chol or its presence at $20 \%$ showed the highest partitioning (Table 2).

Similar $K_{P}$ results were also found by de Granada-Flor et al. ${ }^{12}$ for quercetin interactions with PC (100\%) and other lipid compositions that represented liquid ordered and liquid disordered phases. In this sense, we found that quercetin has a dual affinity pattern on the compositions presenting ld/lo phase coexistence, which depends on their homogeneity level. The apparently controversial behavior in the ld/lo lipid bilayers, in which for $20 \%$ Chol we have the highest $K_{P}$, while at $40 \%$ we have the lowest one, suggested that the homogeneity level should be considered. In fact, there is a high affinity for the lipid composition in the ld phase (PC 100\%), but there is an even greater affinity for the homogeneous composition in a ld/lo phase (PC/Chol 80:20). ${ }^{19}$ These observations are reinforced by the spectral shifts found under the same experimental conditions, which indicate that quercetin migrates from the aqueous environment to the membrane microenvironment more intensely for the most homogeneous compositions. The compositions of lower homogeneity reduce quercetin affinity possibly due to the smaller extension and number of liquid disordered regions where quercetin could interact deeply. Movilenau and coll. ${ }^{8}$ found that quercetin molecules penetrate the DMPC lipid bilayers by intercalating between the flexible acyl chains of the phospholipids depending on the $\mathrm{pH}$ of the medium, and showing that at physiological conditions it translocated to the polar part of the lipid bilayer. The same tendency for quercetin incorporation into lipid membranes was investigated by Kosinova et al. ${ }^{30}$, who showed that quercetin derivatives penetrate the lipid bilayer and that the depths of penetration depend on molecular charge and on the substituent groups in quercetin structure. ${ }^{30}$

Comparing to PC 100\% and PC/Chol 80:20, bilayers with 40\% Chol show a more ordered lipid packing, which is indicated by the results of the anisotropy of the chlorophyll probe, partitioning is reduced and quercetin assumes mostly a more superficial location. This bilayer is also less homogeneous and more rigid than the others and presents nanoscopic lipid domains. ${ }^{13,19}$ 
But, at 20\% Chol and 40\% SM, the bilayer becomes less ordered, more heterogeneous and less rigid than the PC/Chol 60:40, also showing microscopic lipid domains. These features do not affect the partitioning and both present similar $K_{P}$ results. However, the membrane becomes more susceptible to the leakage process. In this sense, inhomogeneity also does not guarantee a unitary mode of action for quercetin. The SM-containing bilayer presents micrometric and probably also nanoscopic domains $(l d+l o+s o)$ and has already been hypothesized to allow for the interdigitation of quercetin molecules, which become defects in the barrier features of the bilayer, facilitating the leakage of internal contents. ${ }^{9}$ 

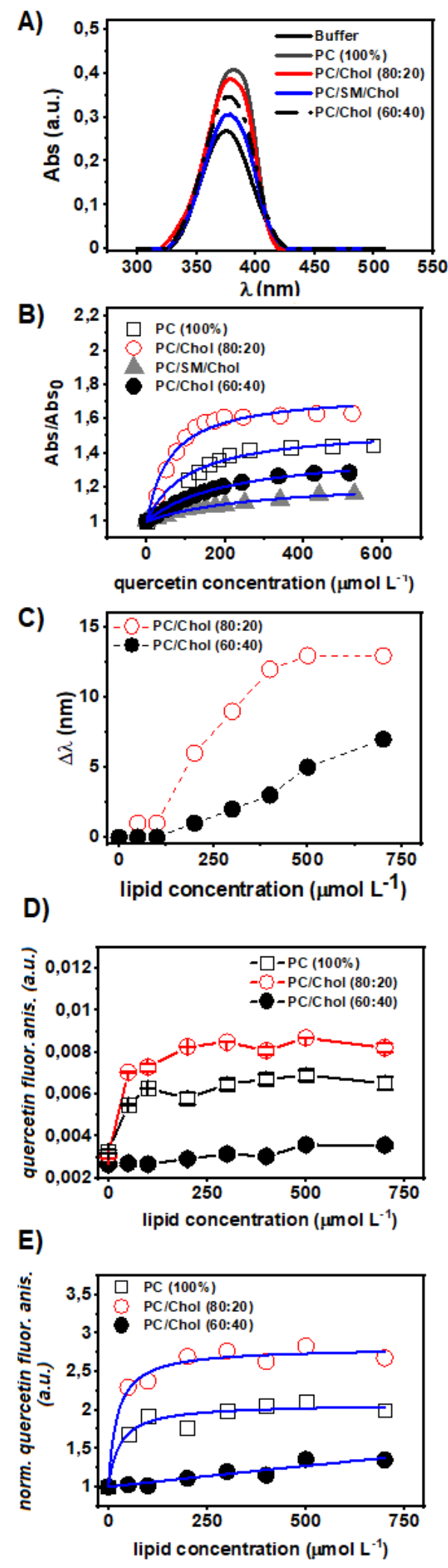

Figure 4. Quercetin partitioning to LUVs of different composition. A) Quercetin $\left(21.5 \mu \mathrm{mol} \mathrm{L}^{-1}\right)$ absorption spectra in the absence $\left(\lambda_{\max }=372 \pm 2 \mathrm{~nm}\right)$ and in the presence of lipid vesicles at 100 
$\mu$ mol L ${ }^{-1}\left(\lambda_{\max }\right.$ values are shown on table S1). B) Wavelength shifts, $\Delta \lambda$, of the maximum spectral absorbance fluorescence for quercetin in the presence of lipid vesicles. C) Partition isotherms obtained from quercetin - lipid spectrophotometric titrations. Changes on quercetin $(21.5 \mu \mathrm{mol} \mathrm{L}-1)$ absorbance, induced by increasing LUVs concentration from 0 to $600 \mu \mathrm{mol} \mathrm{L}{ }^{-1}$ at fixed wavelenght, were obtained and normalized. Solid lines resulted from non-linear fit to Eq. 1 and the obtained $\mathrm{K}_{\mathrm{p}}$ are shown on table 2. D) Quercetin fluorescence anisotropy under LUVs titration. Changes on quercetin fluorescence anisotropy, were obtained under lipid additions ranging from 0 to $700 \mu$ mol $\mathrm{L}^{-1}$. E) Partition isotherms acquired from quercetin - lipid fluorescence anisotropy titrations. Solid lines resulted from non-linear adjust fit to Eq. 2 and the obtained $\mathrm{K}_{\mathrm{p}}$ are shown on table $\mathrm{S} 2$. Experiments were performed at $25^{\circ} \mathrm{C}$ and $\mathrm{pH} 7.0$.

Table 2. Partition Coefficients obtained through Absorbance intensities quercetin as a function of increasing lipid concentrations at distinct lipid compositions.

\begin{tabular}{l|c}
\multicolumn{1}{l}{ LIPID COMPOSITION } & $\boldsymbol{A b s} K_{\boldsymbol{P}}\left(\mathbf{1 0}^{\mathbf{4}}\right)$ \\
\hline PC & $1.00 \pm 0.10$ \\
PC/ Chol (80:20) & $1.60 \pm 0.30$ \\
PC/ SM /Chol (40:40:20) & $0.48 \pm 0.03$ \\
PC/Chol (60:40) & $0.43 \pm 0.03$
\end{tabular}

\section{Experimental}

\subsection{Chemicals}

The following phospholipids, supplied by Avanti Polar Lipids (Alabaster, AL), were used: 1palmitoyl-2-oleoyl-sn-glycero-3-phosphocholine (PC), Sphingomyelin (Egg Chicken, SM), Cholesterol (Chol) and 1,2-dioleoyl-sn-glycero-3-phosphoethanolamine-N-(7-nitro2,1,3benzoxadiazol-4-yl) (ammonium salt) (NBD-PE). Quercetin, TopFluor-Chol and Chlorophyll a were supplied by Sigma-Aldrich Co. (S.Louis, MO). Other chemicals were high-quality analytical or spectroscopic grade. 


\subsection{Quercetin solution and Buffer preparation}

Quercetin solutions were prepared in amber glass vials by diluting $2 \mathrm{mg}$ in $1.5 \mathrm{~mL}$ of $0.6 \%(\mathrm{v} / \mathrm{v})$ dimethylsulfoxide (DMSO) due to its low solubility in pure water. DMSO was kept below 0.6\% (v/v) to avoid effects on the vesicles. The experiments were carried out at $\mathrm{pH} 7$ with buffer made of $10 \mathrm{mM}$ HEPES whose ionic strength was made compatible with the physiological environment by using $150 \mathrm{mM} \mathrm{NaCl}$.

\subsection{Vesicles preparation}

Lipid stock solutions in chloroform were mixed to give the following compositions ( $\mathrm{mol} / \mathrm{mol}$ ): PC (100\%), PC/Chol 80:20, PC/SM/Chol 40:40:20 and PC/Chol 60:40. Mixtures were used to form lipid films through solvent evaporation under nitrogen flux followed by drying under vacuum for 3 h. These samples could additionally contain chlorophyll a for fluorescence anisotropy measurements, or the fluorescent probes NBD-PE (for PC GUVs) or TopFluor-Chol (for Chol containing GUVs) for fluorescence microscopy assays, at $0.5 \mathrm{~mol} \%$ in each case. Suspensions of GUVs (at $1 \mathrm{mg} \mathrm{L}^{-1}$ ) or LUVs (at 5 to $10 \mathrm{mmol} \mathrm{L}^{-1}$ ) were obtained by hydrating these films with 300 mmol $\mathrm{L}^{-1}$ sucrose solution or HEPES buffer, respectively, at room temperature or at $50^{\circ} \mathrm{C}$ for vesicles containing Chol or SM, respectively.

GUVs were prepared by the electroformation method. ${ }^{31}$ For this, $20 \mu \mathrm{L}$ of the desired phospholipid solution were deposited on ITO conductive plates. After solvent evaporation the electroformation chamber was prepared, by using a Teflon spacer and silicone grease, and filled with $1 \mathrm{ml}$ of sucrose solution. The chamber was connected to a generator of alternating current at $10 \mathrm{~Hz}$ and $1 \mathrm{mV}$ for $2 \mathrm{~h}$ at room temperature or at $50^{\circ} \mathrm{C}$ for vesicles containing Chol or SM.

LUVs suspensions were extruded 6 times through $400 \mathrm{~nm}$ polycarbonate membranes and then 11 times through $100 \mathrm{~nm}$ membranes in the Avanti Mini extruder. LUVs were kept under refrigeration, protected from light and used within $24 \mathrm{~h}$ of preparation. LUVs were obtained with an average diameter of 120 to $130 \mathrm{~nm}$ and polydispersity index ranging from 0.09 to 0.15 as confirmed by dynamic light scattering with a Zetasizer NanoZS (Malvern Instruments, Worcestershire, U.K.).

\subsection{Microscopy of GUVs}

Aliquots of GUVs suspension $(1 \mu \mathrm{L})$ were diluted with $10 \mathrm{mM}$ HEPES buffer in a $120 \mu \mathrm{L}$ observation chamber coated with $10 \%$ bovine serum albumin. ${ }^{32}$ To avoid osmotic pressure effects, the osmolarity of the sucrose (inside GUVs) and buffer solution (outside) were checked with an osmometer (Osmette A 5002, Precision Systems, Inc., USA) and carefully matched. The different solvents inside and outside GUVs provided differences in refractive index and density required for observation under the phase contrast microscope (Zeiss Axiocam IC -Zen Lite 2012, Jena, Germany 
equipped with CCD camera) and stabilized GUVs by gravity. After GUVs suspension settled in the chamber, a quercetin solution was added at $21.5 \mu \mathrm{mol} \mathrm{L}{ }^{-1}$. From each lipid composition, at least 20 vesicles with diameters ranging from 20 to $30 \mu \mathrm{m}$ were individually observed for 30 minutes under $40 \times$ phase contrast objective. Vesicles that lost phase contrast induced by quercetin were counted to calculate the number (\%) of these events. Fluorescence images (U-LH100 Hg lamp and 460- 490 $\mathrm{nm}$ excitation filter) were taken to monitor other possible morphological changes during and/or after each observation. In addition, control experiments were performed at each lipid composition in the absence of quercetin through GUVs observation in buffer for a period of 30 minutes.

\subsection{Permeability Estimative}

The set of GUVs observed by phase contrast microscopy on time-lapse mode were analyzed with the help of ImageJ software for imaging analysis that calculate vesicle permeability as a function of quercetin interaction for vesicles that lost phase contrast. Thus, the sucrose leakage profile was obtained from the phase contrast gray-level changes measured along the GUV edge as a function of time for each GUV. The phase contrast average intensity might be evaluated from:

$$
c(t)=\frac{\left(I_{\text {edge }, t}-I_{\text {background }, t}\right)}{\left(I_{\text {edge }, 0}-I_{\text {background }, 0}\right)}
$$

where, $\mathrm{I}_{\text {edge }}$ is relative to maximum average intensity at the edge of the vesicle, $\mathrm{I}_{\text {background }}$ is relative to the background, and sub index 0 is relative to the initial instant of observation. The $\mathrm{c}(\mathrm{t})$ assesses the GUVs permeability induced by quercetin through the linear fit of the slope in the plot $\mathrm{R} / 3 \ln (\mathrm{c}(\mathrm{t}))$ vs $\mathrm{t}$, in which $\mathrm{R}$ is given by the GUV radius. ${ }^{33}$

\subsection{Chlorophyll emission anisotropy}

The fluorescent probe chlorophyll a might be used as a reporter of the phospholipid acyl chain order through emission anisotropy measurements. ${ }^{6}$ LUVs suspension at $50 \mu \mathrm{mol} \mathrm{L}{ }^{-1}$ phospholipid concentration, whose films contained this lipophilic fluorescent probe, were titrated in PMMA cuvettes (Sarstedt ref. 67755) by adding aliquots of the quercetin solution. For these measurements, excitation and emission wavelengths were set at 430 and $678 \mathrm{~nm}$ and bandwidths at $2 \mathrm{~nm}$ and $1 \mathrm{~nm}$, respectively, in an ISS PC1 spectrofluorometer (Urbana Champaign, IL, USA) equipped with GlanThompson polarizers. The excitation and emission pathways were set at $90^{\circ}$ and $0^{\circ}$, respectively, during 21 iterations, at $25^{\circ} \mathrm{C}$. The fluorescence anisotropy $(r)$ was calculated using the equation $\mathrm{r}=$ $(\mathrm{F} \|-\mathrm{GF} \perp) /(\mathrm{F} \|+2 \mathrm{GF} \perp)$, where $\mathrm{F} \|$ is the fluorescence emission intensity parallel to the excitation plane, $\mathrm{F} \perp$ is the normal component to the same plane, and $\mathrm{G}$ is the instrumental factor.

\subsection{Spectrophotometric titrations}

Spectrophotometric titrations were carried out to obtain quercetin partition coefficients, $K_{P}$, at each vesicle composition. Quercetin solutions in buffer were prepared at $21.5 \mu \mathrm{mol} \mathrm{L}{ }^{-1}$ and titrated at 
$25^{\circ} \mathrm{C}$ by the addition of aliquots of LUVs, whose final concentration ranged from 0 to around 600 $\mu \mathrm{mol} \mathrm{L}{ }^{-1}$. PMMA cuvettes (Sarstedt ref. 67755) of $10 \mathrm{~mm}$ path length were used. At each aliquot added, an absorption spectrum was recorded from 300 to $650 \mathrm{~nm}$ with a UV-Vis spectrophotometer Shimadzu UV2600 model (Shimadzu Corp., Japan). Spectra were corrected for dilution effects on quercetin concentration and scattering effects of vesicles. This latter correction was made by titrating the vesicle suspension in the absence of quercetin for each lipid composition. The intensity of maximum absorbance was obtained from each spectrum by the second derivative method ${ }^{34}$ and used to obtain the isotherms curves for $K_{P}$ determination by fitting the plots with:

$$
\frac{A b s(L)}{A b s_{0}}=1+\left(\frac{A b s_{\max }}{A b s_{0}}-1\right) \frac{K p L[L]}{1+K p L[L]}
$$

where $A b s(L), A b s_{0}$ and $A b s_{\max }$ are, respectively, the absorbance in the presence of LUVs, in the absence and the maximum absorbance obtained; $[L]$ is the phospholipid concentration; $K_{P}$ is the molecular lipid/water partition coefficient, and $\gamma_{L}$ the molar volume of the lipid. ${ }^{28}$ Duplicate experiments were performed. A previous experiment in which quercetin in the concentration range of 0 to $25 \mu \mathrm{mol} \mathrm{L}{ }^{-1}$ was titrated into buffer and into vesicles suspensions $\left(100 \mu \mathrm{mol} \mathrm{L}{ }^{-1}\right)$ confirmed that no deviation of the Beer-Lambert law occurred and no signs of insolubility effects appeared.

Quercetin intrinsic fluorescence was used to monitor its emission anisotropy and access the partition coefficients in the different lipid compositions by an alternative method. Excitation and emission wavelength were set at 460 and $540 \mathrm{~nm}$, respectively, with bandwidths of $2 \mathrm{~nm}$, and using a $1 \mathrm{~cm}$ path length quartz cell. Changes on the quercetin anisotropy fluorescence were determined by titrating solutions made of $21.5 \mu \mathrm{mol} \mathrm{L}^{-1}$ flavonoid total concentration with increasing aliquots of LUVs suspension. The experiments were performed in the ISS PC1 spectrofluorometer and the same conditions as detailed in section 2.6. Partition coefficients, $K_{P}$, resulted from the nonlinear fit of the plot $r / r_{0}$ vs lipid concentration according to the expression: ${ }^{28}$

$$
\frac{r}{r_{W}}=\frac{\left(\left(\gamma_{L}[L]\right)^{-1}-1\right)+K_{P} \frac{\left(\varepsilon_{L} \varphi_{L} r_{L}\right.}{\left.\varepsilon_{W} \varphi_{W}\right) r_{W}}}{\left(\gamma_{L}[L]\right)^{-1}-1+K_{P} \frac{\left(\varepsilon_{L} \varphi_{L}\right.}{\left.\varepsilon_{W} \varphi_{W}\right)}}
$$

where $r$ is the average anisotropy, $r_{W}$ is the average anisotropy in buffer, $\varepsilon$ being the molar extinction coefficient, $\varphi$ the quantum yield, $\gamma_{L}$ the lipid molar volume and [L] the lipid concentration. Sub-indexes L and $\mathrm{W}$ represent lipid and aqueous phase, respectively. 


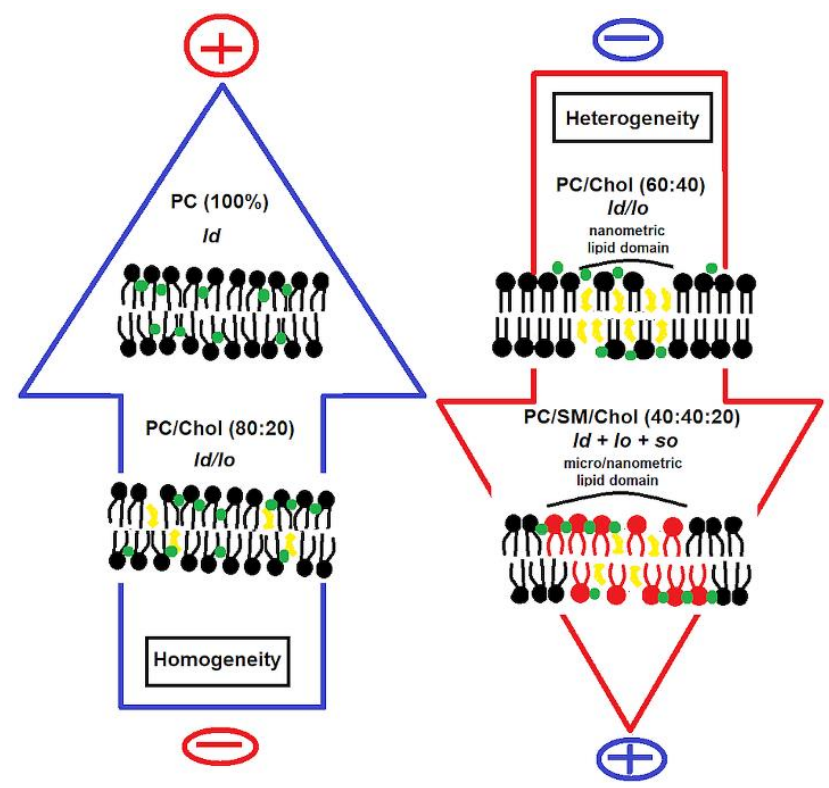

Figure 5. Schematic representation of quercetin interaction with the studied model membranes, where the homogeneity favored partitioning of quercetin molecules and heterogeneity distinctly affected the permeability. Higher heterogeneity with the presence of microdomains and possibly nanodomains increased permeability. Presence of nanoscopic domains decreased membrane permeability. Symbols: black, PC lipids; yellow, Chol; red, SM lipids and green, quercetin molecules.

\section{Conclusions}

Quercetin is a polyphenolic molecule that has diverse/promiscuous biological activities. ${ }^{12}$ The cell membrane concentrates the available quercetin molecules, being a strongly related component of its mechanism of action, and turning the investigation of biophysical properties an important step in advanced studies of quercetin-membrane interaction. Figure 5 illustrates our suggestion for the mechanism of quercetin interaction with different model membranes. We observed that quercetin induces lipid domain-dependent permeability, that is, when high heterogeneity conferred by micrometric lipid domains is present, strong permeability occurs, while in the presence of nanoscopic domains the permeability is refrained. Exploring the interaction of quercetin with model membranes of different lipid compositions is our strategy to access the importance of lipid phases, $l d$, $l d / l o$ and $l d+l o+s o$, to the action of quercetin in bilayers and possibly contribute to the understanding of quercetin multiple activities. The microscopy results showed that the average permeability induced by quercetin in the different lipid bilayers is dependent on the presence of lipid domains. PC/SM/Chol (40:40:20) GUVs exhibited micrometric lipid domains, which coalesce 
during the interaction, and promote average permeability to at least ten times larger levels than observed with the other lipid compositions. In spite of the different heterogeneity of these bilayers, observed in the microscopy studies, and the spectrophotometric data showing that PC/SM/Chol (40:40:20) bilayer is less ordered than PC/Chol 60:40, these different features did not impact the $K p$ values which are similar. In contrast, quercetin showed greater affinity for the PC (100\%) and PC/Chol (80:20) compositions, which are more homogeneous and have greater extension and number of liquid disordered regions. In this sense, inhomogeneity played an important role in the interaction of quercetin with the studied membranes, but it did not guarantee a unique mode of action for quercetin. The presence of SM led to the formation of micrometric and probably also nanoscopic domains corroborating the hypothesis that quercetin molecules interdigitate between lipids, allowing the opening of defects in the lipid bilayer and increasing the permeability of the membrane.

\section{Author Contributions}

The manuscript was written through contributions of all authors. All authors have given approval to the final version of the manuscript.

\section{Conflicts of interest}

The manuscript was written through contributions of all authors. All authors have given approval to the final version of the manuscript.

\section{Acknowledgements}

This research was funded by Fundação de Amparo à Pesquisa do Estado de São Paulo - FAPESP Nos. 2012/24259-0, 2014/08372-7, 2014/06713-1, 2014/11877-3. DBM and VEF are recepients of CAPES scholarships.

The authors are thankful to Prof. Dr. M. C. Bisinoti and A. B. Moreira for the use of the spectrophotometer (FAPESP 2012/23066-4) and Prof. Dr. P. Rahal for the use of the fluorescence microscope. The authors acknowledge the assistance of Mrs. Maira Ramos Vieira and Mr. Vinicius E. Fazani in spectroscopy and microscopy experiments.

\section{Notes}

The authors declare no competing financial interest. 


\section{ABBREVIATIONS}

LUV, large unilamellar vesicle; GUV, giant unilamellar vesicle.

\section{AUTHOR INFORMATION}

\section{Corresponding Author}

*E-mail: natalia.slade@uftm.edu.br. Phone: +55(34) 992632244.

\section{Present Address}

$\dagger$ N.B.L. present address: Departamento de Física, Universidade Federal do Triângulo Mineiro

(UFTM), Instituto de Ciências Naturais, Exatas e Educação (ICENE), Univerdecidade, Uberaba, MG, Brazil.

\section{ORCID}

Natália B. Leite: 0000-0001-5442-1765

Marcia Perez dos Santos Cabrera: 0000-0001-7443-2883

\section{References}

1 S. Teixeira, C. Siquet, C. Alves, I. Boal, M. P. Marques, F. Borges, J. L. Lima and S. Reis, Radic Biol Med, 39, 1099-108;

2 H. Tsushiya, Food Chemistry, 2010, 120, 1089-1096;

3 G. T. Rich, M. Buchweitz, M. S. Winterbone, P. A. Kroon and P. J. Wilde. Nutrients, 2017, 9, 111;

4 F. Vargas, P. Romecín, A. I. García-Guillén, R. Wangesteen, P. Vargas-Tendero, M. D. Paredes, N. M. Atucha and J. García-Estañ, Front. Physiol., 2018, 9, 394;

5 C. Carrasco-Pozo, K. N. Tan, M. Reyes-Farias, N. De La Jara, S. Thieu Ngo, D. F. GarciaDiaz, P. Llanos, M. J. Cires and K. Borgese, Redox Biology,2016, 9, 229-243;

6 M. Drăguşin, L. Ţugulea and C. Ganea, Gen. Physiol. Biophys. J. 2010, 29, 41-49;

7 F. Ollila, K. Halling,P. Vuorela, H. Vuorela and J. P. Slotte, Archives of Biochemistry and Biophysics, 2002, 399(1), 103-108; 
8 L. Movileanu, I. Neagoe and M. L. Flonta, International Journal of Pharmaceutics, 2000, 205(1-2), 135-146;

9 Y. S. Tarahovsky, E. N. Muzafarov and Y. A. Kim, Mol Cell Biochem, 2008, 314, 65-71;

10 N.P. Ulrih, M. Maričić, A. Ota, M. Šentjurc and V. Abram, Food Research International, 2015, 71, 146-154;

11 N. B. Leite, D. B. Martins, V. E. Fazzani, M. R. Vieira and M. P. dos Santos Cabrera, Biochimica et Biophysica Acta (BBA) - Biomembranes, 2018, 1860(11), 2320-2328;

12 A. de Granada-Flor, C. Sousa, H. A. L. Filipe, M. Santos and R. F. M. de Almeida, Chem Commun (Camb), 2019, 55(12),1750-3;

13 F. M. Goñi, A. Alonso, L. A. Bagatolli, R. E. Brown, D. Marsh, M. Prieto and J. L. Thewalt, Biochimica et Biophysica Acta., 2008, 1781(11-12), 665-684;

14 D. Marsh, Biochimica et Biophysica Acta (BBA) - Biomembranes, 2009, 1788(10), 21142123

15 M.V. Lizenko, T.I. Regerand, A.M. Bakhirev and E.I. Lizenko, J. Evol. Biochem. Physiol., 2011, 47, 428-437;

16 T. Harayama and H. Riezman, Nature Reviews Molecular Cell Biology, 2018, 19(5), 281296;

17 R.F. de Almeida, A. Fedorov and M. Prieto, Biophys. J., 2003, 85, 2406-2416;

18 R. Dimova, Annual Review of Biophysics, 2020, 48, 93 - 119;

19 N. K. Sarangi, K. G. Ayappa and J. K. Basu, Scientific Reports, 2017, 7(1), 11173;

20 F. Schmid, Biochimica et Biophysica Acta (BBA) - Biomembranes, 2017, 1859(4), 509-528;

21 J. D. Nickels, X. Cheng, B. Mostofian, C. Stanley, B. Lindner, F. A. Heberle and J. Katsaras, Journal of the American Chemical Society, 2015, 137(50), 15772-15780;

22 F. A. Heberle, M. Doktorova, H. L. Scott, A. Skinkle, M. N. Waxham and I. Levental, bioRxiv, 2020, 117(33), 19943-19952; 
23 C. M. Rosetti, A. Mangiarotti and N. Wilke, Biochim Biophys Acta Biomembr, 2017, 1859(5),789-802;

24 J .R. Lakowicz, Springer, 3rd edition , 2006;

25 A. Filippov, G. Orädd and G. Lindblom, Langmuir, 2003, 19, 16, 6397-6400;

26 H.I. Ingólfssson, P. Thakur, K.F. Herold, E.A. Hobart, N.B. Ramsey, X. Periole, D.H. de Jong, M. Zwama, D. Yilmaz, K. Hall, T. Maretzky, H.C. Hemmings Jr., C. Blobel, S.J. Marrink, A. Koçer, J.T. Sack and O.S. Andersen, ACS Chem. Biol., 2014, 9, 1788-1798;

27 R. Sinha, M. K. Gadhwal, U. J. Joshi, S. SrivaStava and G. Govil, J Indian Chem Soc, 2011, 88, 1203-1210;

28 N. C. Santos, M. Prieto and M. A. R. B Castanho, Biochim. Biophys. Acta, 2003, 1612, 123135

29 D. Patra, E. El Khoury, D. Ahmadieh, S. Darwish and R. M. Tafech, Photochem. Photobiol., 2012, 88, 317-327;

30 P. Košinová, K. Berka, M. Wykes, M. Otyepka and P. Trouillas, The Journal of Physical Chemistry B, 2012, 116(4), 1309-1318;

31 M. I. Angelova and D. S. Dimitrov, Faraday Discuss. Chem. Soc, 1986, 81, 303-311;

32 S. Ritz, K. Eisele, J. Dorn, S. Ding, D. Vollmer, S. Putz, T. Weil and E. K. Sinner, et al., Biointerphases, 2010, 5, FA78-FA87;

33 D. S. Alvares, T.G. Viegas and J. Ruggiero Neto, Chemistry and Physics of Lipids, 2018, 216, 54-64;

34 H. I. Ingólfsson, R. E. Koeppe and O. S. Andersen, Biochem. 2007, 46, 10384-10391. 